
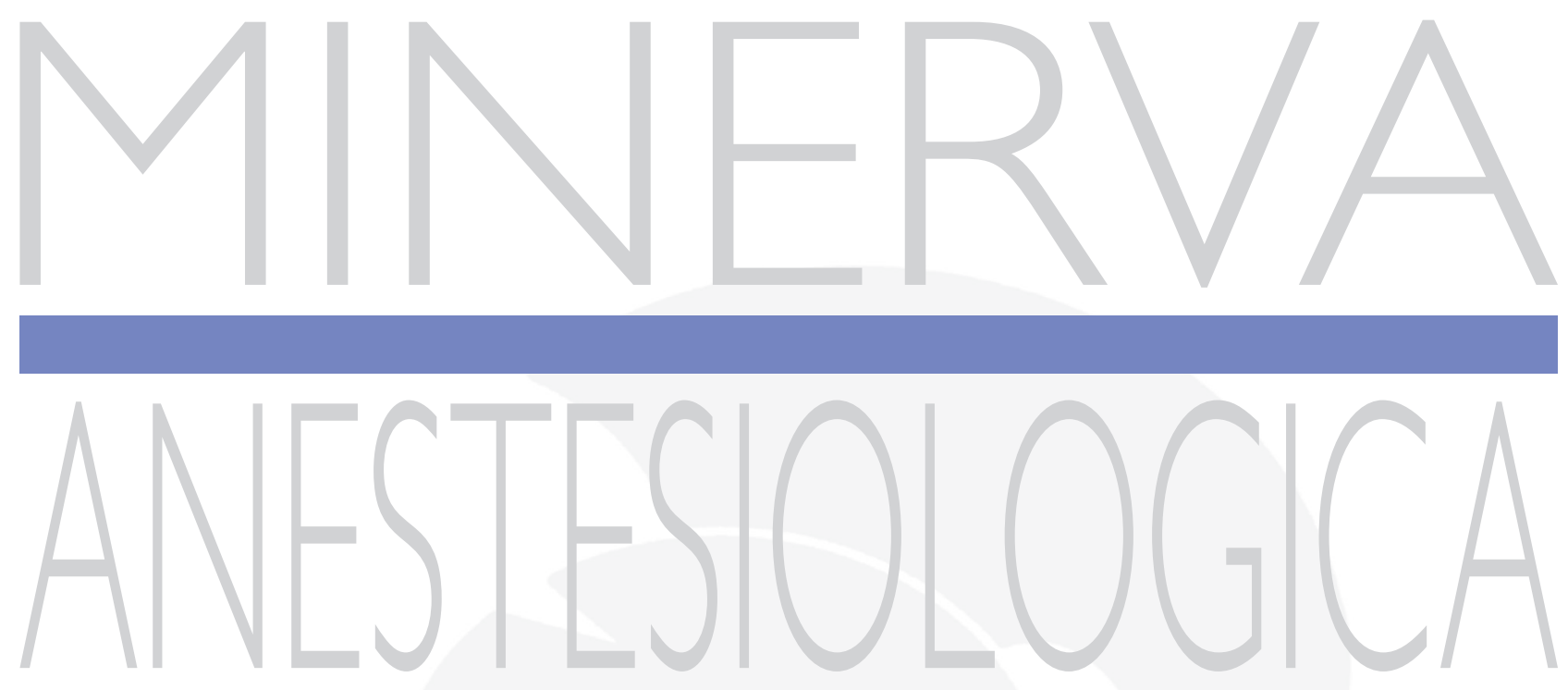

OFFICIAL JOURNAL OF ITALIAN SOCIETY OF ANESTHESIOLOGY, ANALGESIA, RESUSCITATION AND INTENSIVE CARE (SIAARTI)

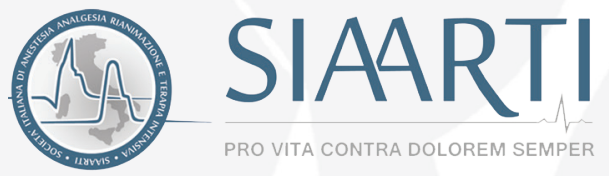

VOLUME $85 \cdot$ No. 5. MAY 2019

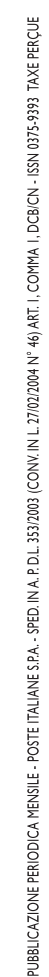

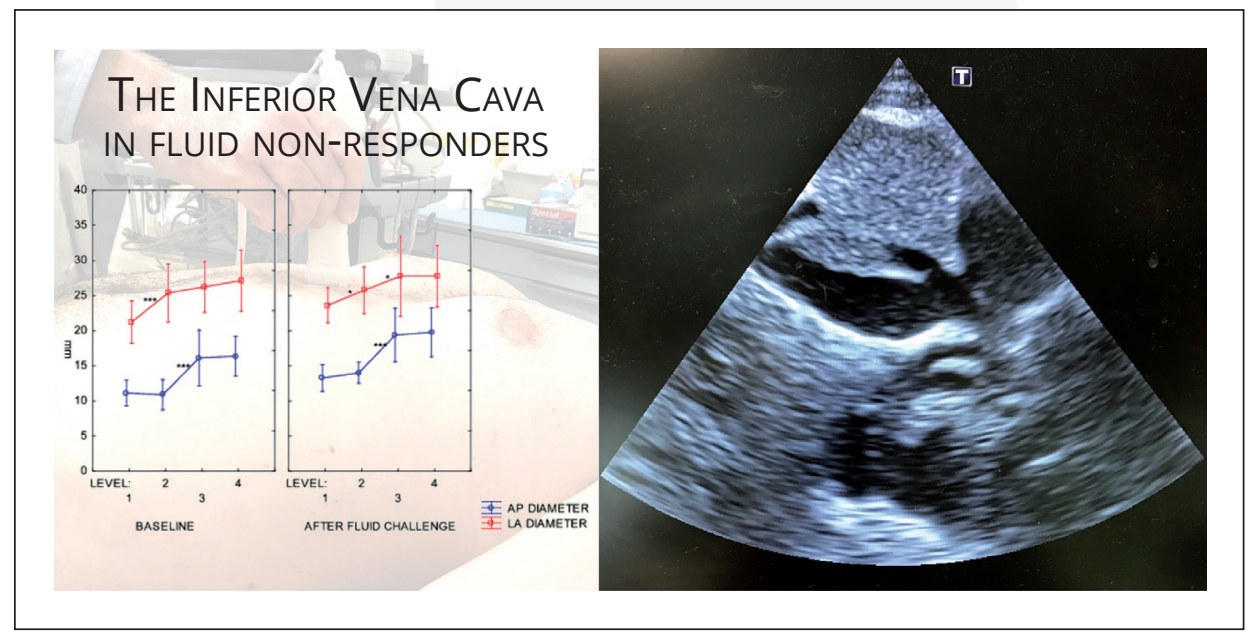

$E D|Z| O N|\cdot M| N E R V A \cdot M E D \mid C A$ 


\title{
Intraoperative nociception: "if you can't measure it, you can't manage it"
}

\author{
Flaminia COLUZZI *
}

Department of Medical and Surgical Sciences and Biotechnologies, Sapienza University, Rome, Italy

*Corresponding author: Flaminia Coluzzi, Department of Medical and Surgical Sciences and Biotechnologies, Sapienza University, Rome, Italy. E-mail: flaminia.coluzzi@uniroma1.it

$\mathrm{M}$ odern anesthesia is based on "numbers" that reflect different clinical parameters and their monitoring over time. The well-known economist Peter Druker, described as the founder of modern management, is credited with a famous quote: "if you can't measure it, you can't manage it." This piece perfectly fits with anesthesia, where monitoring clinical data is essential for adequate patient management.

Before the advent of modern anesthesia, "painless surgery" was just a utopia; therefore, the first objective of anesthesia was analgesia and it is still today. General anesthesia is essentially a combination of three components: analgesia, hypnosis, and muscle relaxation. However current clinical practice includes routine use of devices for monitoring depth of anesthesia and for the measurement of neuromuscular block, while monitoring intraoperative nociception is still a step backward in its evolution. ${ }^{1}$

Pain pathway is a complex mechanism, probably still not completely known, where spinal and supra-spinal phenomena contribute to transmission and perception. Monitoring suppression of nociception during surgery could be essential for tailoring opioid administration, improving postoperative pain, and preventing hyperalgesia. Limiting opioid use during surgery has become a main target in modern anesthesia, with a growing role for the regional techniques and an incoming space for total opioid-free techniques, particularly in vulnerable patients. ${ }^{2}$ Anesthesia should be administered using appropriate doses, with the aims of enhancing recovery and facilitating early mobilization. ${ }^{3}$ However, in clinical practice, analgesia is still empirically monitored by using variation of heart rate, blood pressure, and sweating, as the result of the sympathetic activation, or movements. ${ }^{4}$

In this issue of Minerva Anestesiologica, Jiao et al. performed a meta-analysis of current available trials evaluating intraoperative nociceptive monitoring as a guide for opioid administration during surgery. ${ }^{5}$ Authors evaluated three devices: Analgesia Nociception Index (ANI; MDMS, Loos, France), Surgical Pleth Index (SPI; GE Healthcare, Helsinki, Finland) and pupillometry.

The ANI is a parameter that provides an estimation of the balance between parasympathetic and sympathetic outflow. The ANI measures heart rate variability from ECG monitoring and, through an algorithm, provides a number from 0 to 100: 0 means a high parasympathetic modulation, and therefore a low stress level, while 100 means a low parasympathetic modulation, and therefore a high stress level. ${ }^{6}$ The SPI is a normalized score that reflects the activation of the parasympathetic system by noxious stimulation. SPI is derived by heart rate and pulse wave amplitude, varying from 0 to 100 , where high values indicate high level of nociception. ${ }^{7}$ Despite the potential benefits of these monitoring systems, there are still many limitations. Severe arrhythmias, atrial fibrillation, and implanted pacemak- 
ers affect the use of the R-R interval as a reliable value of sympathetic-parasympathetic balance. Moreover, the use of antimuscarinic drugs modify the assessment of the antinociception/nociception balance.

All these monitoring systems are based on the concept that nociception induces a linear stress response, detectable through physiological parameters. This dogma may be not correct, as recently demonstrated by Ledowski et al., which showed that cathecolamine plasma levels and hemodynamic parameters did not correlate as expected with postoperative pain. ${ }^{8}$

Pupillary diameter increases in response to nociceptive stimuli and varies during surgery according to surgical stimuli and opioid administration. This "pupillary reflex dilation" can be recorded with the video pupillometer Algiscan (IDMed, Marseille, France), through an infrared camera that, without touching the eyes, recognizes and tracks the pupil size. This system has been used for guiding remifentanil administration during total intravenous anesthesia. ${ }^{9}$ Unfortunately, even this system has some limitations, including the influence of ambient light on pupillary diameter and the lack of data in other types of anesthesia.

The challenge of monitoring analgesia in anesthetized patients is related to the interference of hypnosis and cardiovascular drugs used during general anesthesia, and to the rapid changes of the sympathetic system activation observed during surgery. Vagal hypertone or sympathetic activity, which occur during general anesthesia or due to the surgical procedure, such as during pneumoperitoneum, may change hemodynamic parameters and affect the reliability of nociception monitoring.

When comparing intraoperative nociception monitoring with the standard clinical practice different outcomes can be used: intraoperative opioid consumption, extubation time, postoperative pain score, and perioperative adverse events, such as hemodynamic events or postoperative nausea and vomiting (PONV). ${ }^{10}$

Previous studies showed that the ANI, the SPI, and pupillary dilatation were superior to clinical signs in detecting changes in the antinociception nociception balance in patients undergoing total intravenous anesthesia. ${ }^{11}$ According to Jiao et al. SPI was more effective on intraoperative analgesia than standard of care in adults, while ANI failed to show any advantage. However, the reduction of intraoperative opioid consumption was significant only during sevoflurane anesthesia. ${ }^{5}$

In conclusion, there are still too many questions about the clinical use of these devices with the aim to give specific recommendations. Firstly, clinicians need to know the clinically relevant thresholds of each system, which consent to discriminate effective versus insufficient analgesia. Consensus on these data is still lacking and further studies are warranted to identify the real meaning of the recorded numbers. Secondly nociception monitoring systems seem to have been commercialized with few studies investigating the potential clinical benefits associated with their use. Most studies are not double blinded, with reduced quality of the trial design. Therefore, expectations may be higher compared with their real potential, and clinicians may have the perception of poor utility or inefficacy. Thirdly, while the intraoperative doses of opioids seem to be significantly reduced by using nociception monitoring, with regard to postoperative pain intensity and relative opioid consumption, these systems did not show any advantage over standard of care. ${ }^{5}$

Therefore, I can conclude that, as attributed to Albert Einstein, "not everything that can be counted counts, and not everything that counts can be counted." The role of currently available devices for intraoperative nociception monitoring is still unclear, but, for adequate analgesia management during surgery, the need for an objective measurement as an alternative to the current clinical standard of care is imminent.

\section{References}

1. Constant I, Sabourdin N. Monitoring depth of anesthesia: from consciousness to nociception. A window on subcortical brain activity. Paediatr Anaesth 2015;25:73-82.

2. Sultana A, Torres D, Schumann R. Special indications for Opioid Free Anaesthesia and Analgesia, patient and procedure related: including obesity, sleep apnoea, chronic obstructive pulmonary disease, complex regional pain syndromes, opioid addiction and cancer surgery. Best Pract Res Clin Anaesthesiol 2017;31:547-60.

3. White SM, Altermatt F, Barry J, Ben-David B, Coburn M, 
Coluzzi F, et al. International Fragility Fracture Network Delphi consensus statement on the principles of anaesthesia for patients with hip fracture. Anaesthesia 2018;73:863-74.

4. Cividjian A, Petitjeans F, Liu N, Ghignone M, de Kock M, Quintin L. Do we feel pain during anesthesia? A critical review on surgery-evoked circulatory changes and pain perception. Best Pract Res Clin Anaesthesiol 2017;31:445-67.

5. Jiao Y, He B, Tong X, Xia R, Zhang C, Shi X. Intraoperative monitoring of nociception for opioid administration: a meta-analysis of randomized controlled trials. Minerva Anestesiol 2019;85:522-30.

6. Daccache G, Jeanne M, Fletcher D. The Analgesia Nociception Index: Tailoring Opioid Administration. Anesth Analg 2017;125:15-7.

7. Ledowski T, Burke J, Hruby J. Surgical pleth index: prediction of postoperative pain and influence of arousal. Br J Anaesth 2016;117:371-4.
8. Ledowski T, Reimer M, Chavez V, Kapoor V, Wenk M Effects of acute postoperative pain on catecholamine plasma levels, hemodynamic parameters, and cardiac autonomic control. Pain 2012;153:759-64.

9. Sabourdin N, Barrois J, Louvet N, Rigouzzo A, Guye ML, Dadure C, et al. Pupillometry-guided Intraoperative Remifentanil Administration versus Standard Practice Influences Opioid Use: A Randomized Study. Anesthesiology 2017;127:284-92.

10. Gruenewald M, Dempfle A. Analgesia/nociception monitoring for opioid guidance: meta-analysis of randomized clinical trials. Minerva Anestesiol 2017;83:200-13.

11. Funcke S, Sauerlaender S, Pinnschmidt HO, Saugel B, Bremer K, Reuter DA, et al. Validation of Innovative Techniques for Monitoring Nociception during General Anesthesia: A Clinical Study Using Tetanic and Intracutaneous Electrical Stimulation. Anesthesiology 2017;127:272-83.

Conflicts of interest.-The authors certify that there is no conflict of interest with any financial organization regarding the material discussed in the manuscript.

Comment on: Jiao Y, He B, Tong X, Xia R, Zhang C, Shi X. Intraoperative monitoring of nociception for opioid administration: a meta-analysis of randomized controlled trials. Minerva Anestesiol 2019;85:522-30. DOI: 10.23736/S0375-9393.19.13151-3

Article first published online: February 13, 2019. - Manuscript accepted: February 5, 2019. - Manuscript received: January $22,2019$.

(Cite this article as: Coluzzi F. Intraoperative nociception: "if you can't measure it, you can't manage it". Minerva Anestesiol 2019;85:462-4. DOI: 10.23736/S0375-9393.19.13612-7) 